\title{
Accelerated Overrelaxation Method
}

\author{
By Apostolos Hadjidimos
}

\begin{abstract}
This paper describes a method for the numerical solution of linear systems of equations. The method is a two-parameter generalization of the Successive Overrelaxation (SOR) method such that when the two parameters involved are equal it coincides with the SOR method. Finally, a numerical example is given to show the superiority of the new method.
\end{abstract}

1. Introduction. For the numerical solution of linear systems, numerous direct as well as indirect methods exist. Among the indirect or iterative methods the Successive Overrelaxation (SOR) and related methods play a very important role and are the most popular ones. These methods are fully covered in the excellent books by Varga [1], by Wachspress [2] and in the most recent one by Young [3] .

The purpose of this paper is to present a two-parameter generalization of the SOR method and also the first basic results concerning this method which has been called Accelerated Overrelaxation (AOR) method. As will be seen, the well-known methods of Jacobi, of Gauss-Seidel, of Simultaneous Overrelaxation and of Successive Overrelaxation can be derived, as special cases, from the AOR method. Finally a characteristic numerical example, which we give in a special case, shows the superiority of the AOR method.

2. Derivation of the AOR Method. We consider a system of $N$ linear equations with $N$ unknowns written in matrix form

$$
A x=b,
$$

where the matrix $A$ has nonvanishing diagonal elements. We also consider the following splitting of $A$

$$
A \equiv D-A_{L}-A_{U},
$$

where $D$ is a diagonal matrix and $A_{L}$ and $A_{U}$ are strictly lower and upper triangular matrices, respectively.

For the numerical solution of Eq. (2.1) we propose to use the most general linear stationary iterative scheme whose matrix coefficients are linear functions of the components of $\boldsymbol{A}$ and the coefficient of the new iterate is an at most lower triangular matrix. This scheme must be of the form

$$
\left(\alpha_{1} D+\alpha_{2} A_{L}\right) x^{(n+1)}=\left(\alpha_{3} D+\alpha_{4} A_{L}+\alpha_{5} A_{U}\right) x^{(n)}+\alpha_{6} b \mid n=0,1,2, \ldots,
$$


where $\alpha_{i} \mid i=1(1) 6$ are constants to be determined $\left(\alpha_{1} \neq 0\right)$ and $x^{(0)}$ an arbitrary initial approximation to the solution $x$ of (2.1). By dividing through by $\alpha_{1}$ we obtain

$$
\left(D+\alpha_{2}^{\prime} A_{L}\right) x^{(n+1)}=\left(\alpha_{3}^{\prime} D+\alpha_{4}^{\prime} A_{L}+\alpha_{5}^{\prime} A_{U}\right) x^{(n)}+\alpha_{6}^{\prime} b \mid n=0,1,2, \ldots,
$$

where we have set $\alpha_{i}^{\prime}=\alpha_{i} / \alpha_{1} \mid i=2(1) 6$. Sufficient conditions for scheme (2.3) to be consistent with Eq. (2.1) are

$$
\left(1-\alpha_{3}^{\prime}\right) D+\left(\alpha_{2}^{\prime}-\alpha_{4}^{\prime}\right) A_{L}-\alpha_{5}^{\prime} A_{U} \equiv \alpha_{6}^{\prime} A, \alpha_{6}^{\prime} \neq 0
$$

In view of (2.2), the first relationship of (2.4) gives

$$
1-\alpha_{3}^{\prime}=\alpha_{6}^{\prime}, \quad \alpha_{2}^{\prime}-\alpha_{4}^{\prime}=-\alpha_{6}^{\prime} \text { and }-\alpha_{5}^{\prime}=-\alpha_{6}^{\prime} .
$$

The above set of equations has the following two-parameter solution

$$
\alpha_{2}^{\prime}=-r, \quad \alpha_{3}^{\prime}=1-\omega, \quad \alpha_{4}^{\prime}=\omega-r, \quad \alpha_{5}^{\prime}=\omega \quad \text { and } \quad \alpha_{6}^{\prime}=\omega,
$$

where $r$ and $\omega \neq 0$ are any two fixed parameters. Consequently, (2.3) becomes

$$
(I-r L) x^{(n+1)}=[(1-\omega) I+(\omega-r) L+\omega U] x^{(n)}+\omega c \mid n=0,1,2, \ldots
$$

where we have set

$$
L=D^{-1} A_{L}, \quad U=D^{-1} A_{U}, \quad c=D^{-1} b
$$

and $I$ the identity matrix of order $N$. Method (2.5) is what is called from now on Accelerated Overrelaxation (AOR) method or $M_{r, \omega}$-method. We observe that for specific values of the parameters $r$ and $\omega$, the $M_{r, \omega}$-method reduces to well-known methods. Thus

$M_{0,1}$-method is the Jacobi method,

$M_{1,1}$-method is the Gauss-Seidel method,

$M_{0, \omega}$-method is the Simultaneous Overrelaxation method, and

$M_{\omega, \omega}$-method is the Successive Overrelaxation method.

From now on we shall call $r$ the acceleration parameter and $\omega$ the overrelaxation parameter and shall use the notations $L_{r, \omega}$ for the iterative matrix of scheme (2.5) given by

$$
L_{r, \omega}=(I-r L)^{-1}[(1-\omega) I+(\omega-r) L+\omega U]
$$

and $\rho\left(L_{r, \omega}\right)$ for the spectral radius of $L_{r, \omega}$.

It should be noted that, except for the case $r=0$, the AOR method is essentially the Extrapolated (E)SOR method with overrelaxation parameter $r$ and extrapolation one $s=\omega / r$, for it is easy to show that

$$
L_{r, \omega}=s L_{r, r}+(1-s) I .
$$

Thus if $\nu$ is an eigenvalue of $L_{r, r}(r \neq 0)$ and $\lambda$, the corresponding one of $L_{r, \omega}$, we have that

$$
\lambda=s \nu+(1-s)
$$


In what follows we shall try to find, under various assumptions on the original matrix $A$, the restrictions imposed on the parameters $r$ and $\omega$ so that the $M_{r, \omega}$-method converges.

3. Irreducible Matrices with Weak Diagonal Dominance. If $\boldsymbol{A}$ is an irreducible matrix with weak diagonal dominance, then it will be nonsingular with nonvanishing diagonal elements. In such a case the following theorem can be proved.

THEOREM. If $A$ is an irreducible matrix with weak diagonal dominance, then the $M_{r, \omega}$-method converges for all $0 \leqslant r \leqslant 1$ and $0<\omega \leqslant 1$.

Proof. We assume that for some eigenvalue $\lambda$ of $L_{r, \omega}$ we have $|\lambda| \geqslant 1$. For this eigenvalue the relationship below holds

$$
\operatorname{det}\left(L_{r, \omega}-\lambda\right)=0
$$

or after performing a simple series of transformations

$$
\operatorname{det}(Q)=0
$$

where we have set

$$
Q=I-\frac{r(\lambda-1)+\omega}{\lambda-1+\omega} L-\frac{\omega}{\lambda-1+\omega} U
$$

The coefficients of $L$ and $U$ in (3.3) are less than one in modulus. To prove this it is sufficient and necessary to prove that

$$
|\lambda-1+\omega| \geqslant|r(\lambda-1)+\omega| \text { and }|\lambda-1+\omega| \geqslant|\omega| .
$$

If $\lambda^{-1}=q e^{i \theta}$ where $q$ and $\theta$ are real with $0<q \leqslant 1$, then the first inequality in (3.4) is equivalent to

$$
\left(1-r^{2}\right)+\left(1-r^{2}\right) q^{2}-\left(1-r^{2}\right) 2 q \cos \theta+(1-r) 2 q \omega \cos \theta-(1-r) 2 q^{2} \omega \geqslant 0,
$$

which holds for $r=1$; otherwise it is equivalent to

$$
(1+r)+(1+r) q^{2}-[(1+r)-\omega] 2 q \cos \theta-2 q^{2} \omega \geqslant 0 .
$$

Since the expression in the brackets above is nonnegative, (3.5) holds for all real $\theta$ if and only if it holds for $\cos \theta=1$. Thus, (3.5) is equivalent to

$$
(1-q)[(1+r)(1-q)+2 q \omega] \geqslant 0
$$

which is true. The second inequality in (3.4) is equivalent to

$$
1+q^{2}-2 q(1-\omega) \cos \theta-2 q^{2} \omega \geqslant 0
$$

which, for the same reason, must be satisfied for $\cos \theta=1$. Thus, we have $(1-q)[(1-q)+2 q \omega] \geqslant 0$ which is also true. Since $A$ has weak diagonal dominance and is irreducible it is obvious that $D^{-1} A=I-L-U$ possesses the same properties. The same is true for the matrix $Q$ since the coefficients of $L$ and $U$ are different from zero and less than one in modulus. Thus, $Q$ is nonsingular which contradicts (3.2) and, consequently, (3.1). Therefore $\rho\left(L_{r, \omega}\right)<1$. 
Considering now the $M_{r, \omega}$-methods corresponding to the pairs $(r, \omega)=(0,1)$, $(1,1),(0, \omega)$ and $(\omega, \omega)$ we can obtain, as a corollary of the previous theorem, the following well-known statement.

COROLlary. If $A$ is an irreducible matrix with weak diagonal dominance, then the methods of Jacobi, of Gauss-Seidel, of Simultaneous Overrelaxation and of Successive Overrelaxation (the last two methods for $0<\omega \leqslant 1$ ), converge.

4. $L$-Matrices. If $A$ is an $L$-matrix that is a matrix whose elements $a_{i j} \mid i, j=$ 1(1) $N$ satisfy the relationships

$$
a_{i i}>0 \mid i=1(1) N \text { and } a_{i j} \leqslant 0 \mid i \neq j, i, j=1(1) N,
$$

then the following theorem concerning the AOR method can be proved.

THEOREM. If $A$ is an L-matrix, then for all $r$ and $\omega$ such that $0 \leqslant r \leqslant \omega \leqslant 1$ $(\omega \neq 0)$ the $M_{r, \omega}$-method converges if and only if the $M_{0,1}$-method converges.

Proof. It is obvious that if the $M_{r, \omega}$-method converges so does the $M_{0,1}$-method. Assume now that $\bar{\lambda}=\rho\left(L_{r, \omega}\right) \geqslant 1$. Because of our assumptions we easily get that $(1-\omega) I+(\omega-r) L+\omega U \geqslant 0$ and also that $(I-r L)^{-1}=I+r L+r^{2} L^{2}+\cdots+$ $r^{N-1} L^{N-1} \geqslant 0$. Thus, for the iterative matrix we have that

$$
L_{r, \omega}=(I-r L)^{-1}[(1-\omega) I+(\omega-r) L+\omega U] \geqslant 0 .
$$

Since $L_{r, \omega}$ is a nonnegative matrix, $\bar{\lambda}$ is an eigenvalue of $L_{r, \omega}$. If $v \neq 0$ is the corresponding eigenvector, we have $L_{r, \omega} v=\bar{\lambda} v$ from which we obtain after some simple manipulation that

$$
\left(\frac{\omega-r+r \bar{\lambda}}{\omega} L+U\right) v=\frac{\bar{\lambda}-1+\omega}{\omega} v
$$

This implies that $(\bar{\lambda}-1+\omega) / \omega$ is an eigenvalue of $((\omega-r+r \bar{\lambda}) / \omega) L+U$. Therefore,

$$
\frac{\bar{\lambda}-1+\omega}{\omega} \leqslant \rho\left(\frac{\omega-r+r \bar{\lambda}}{\omega} L+U\right) .
$$

It is obvious that $(\omega-r+r \bar{\lambda}) / \omega \geqslant 1$ so that

$$
0 \leqslant \frac{\omega-r+r \bar{\lambda}}{\omega} L+U \leqslant \frac{\omega-r+r \bar{\lambda}}{\omega}(L+U)=\frac{\omega-r+r \bar{\lambda}}{\omega} L_{0,1} .
$$

Relationships (4.1) and (4.2) imply that $\bar{\lambda}-1+\omega \leqslant(\omega-r+r \bar{\lambda}) \rho\left(L_{0,1}\right)$ from which we can easily obtain that $\rho\left(L_{0,1}\right) \geqslant 1$. Since we have proved that if $\bar{\lambda} \geqslant 1$, then $\rho\left(L_{0,1}\right) \geqslant 1$, we can readily obtain that $\rho\left(L_{0,1}\right)<1$ implies $\bar{\lambda}<1$ so that if the $M_{0,1}$-method converges then so does the $M_{r, \omega}$-method.

5. Consistently Ordered Matrices. In this section we assume that matrix $A$ is a consistently ordered one, that is, a matrix for which the expression $\operatorname{det}\left(\alpha A_{L}+\alpha^{-1} A_{U}-\beta D\right)$ is independent of $\alpha$ for $\alpha \neq 0$ and for all $\beta$. As can be easily found out, the analysis of this section also applies in the case where $A$ is a matrix which has property $A$. 
Before we begin our analysis we give three lemmas which will be very useful in the sequel.

LEMmA 1. If $A$ is a consistently ordered matrix with nonvanishing diagonal elements, and if $\mu \neq 0$ is an eigenvalue of $L_{0,1}$ of multiplicity $p$, then $-\mu$ is also an eigenvalue of $L_{0,1}$ of multiplicity $p$.

Proof. See Theorems 3.4 and 2.2 on pages 147 and 142, respectively, of Young [3].

LEMMA 2. If $A$ is a consistently ordered matrix with nonvanishing diagonal elements, and if $\mu$ is an eigenvalue of $L_{0,1}$ and $\nu$ satisfies

$$
(\nu-1+r)^{2}=r^{2} \mu^{2} \nu,
$$

then $\nu$ is an eigenvalue of $L_{r, r}$ and vice versa.

Proof. See the same Theorems as in Lemma 1.

LEMmA 3. If $\beta$ and $\gamma$ are real, then both roots of the quadratic equation $\lambda^{2}-$ $\beta \lambda+\gamma=0$ are less than one in modulus if and only if.

$$
|\gamma|<1, \quad|\beta|<1+\gamma .
$$

Proof. See Lemma 2.1, p. 171 of Young [3].

Having stated the three lemmas above, we give in the sequel three theorems concerning the AOR method.

THEOREM 1. If $A$ is a consistently ordered matrix with nonvanishing diagonal elements, and if $\mu$ is an eigenvalue of $L_{0,1}$ and $\lambda$ satisfies

$$
(\lambda-1+\omega)^{2}=\omega \mu^{2}[r(\lambda-1)+\omega],
$$

then $\lambda$ is an eigenvalue of $L_{r, \omega}$ and vice versa.

Proof. Since the requirements of Lemma 2 are fulfilled, for $r \neq 0$, we substitute the value of $\nu$ in terms of $\lambda$ from (2.7) into (5.1) and its equivalent relationship (5.3) follows. For $r=0$ it is easy to show that, by virtue of Lemma $1, \lambda$ satisfies (5.3) and vice versa.

THEOREM 2. If $A$ is a consistently ordered matrix with nonvanishing diagonal elements and if $L_{0,1}$ has real eigenvalues $\mu_{i} \mid i=1(1) N$, with $\underline{\mu}=\min _{i}\left|\mu_{i}\right|$ and $\bar{\mu}=$ $\max _{i}\left|\mu_{i}\right|$, then the $M_{r, \omega}$-method converges if and only if the $M_{0,1}$-method converges and the parameters $\omega$ and $r$ take values from the intervals $I_{\omega}$ and $I_{r}$, respectively, defined as follows

$$
\begin{gathered}
\text { for } \underline{\mu} \neq 0: \quad I_{\omega} \equiv\left(-2 /\left(1-\underline{\mu}^{2}\right)^{1 / 2}, 0\right) \quad \text { and } I_{r} \equiv\left(\beta\left(\underline{\mu}^{2}\right), \alpha\left(\bar{\mu}^{2}\right)\right) \text { or } \\
I_{\omega} \equiv(0,2] \quad \text { and } I_{r} \equiv\left(\alpha\left(\bar{\mu}^{2}\right), \beta\left(\bar{\mu}^{2}\right)\right) \text { or } \\
I_{\omega} \equiv\left[2,2 /\left(1-\underline{\mu}^{2}\right)^{1 / 2}\right) \quad \text { and } I_{r} \equiv\left(\alpha\left(\bar{\mu}^{2}\right), \beta\left(\underline{\mu}^{2}\right)\right) \text { while } \\
\text { for } \underline{\mu}=0: \quad I_{\omega} \equiv(0,2) \text { and } I_{r} \equiv\left(\alpha\left(\bar{\mu}^{2}\right), \beta\left(\bar{\mu}^{2}\right)\right),
\end{gathered}
$$

where 


$$
\alpha(z) \equiv \frac{1}{\omega z}\left(\frac{1}{2} \omega^{2} z-\frac{1}{2} \omega^{2}+2 \omega-2\right) \quad \text { and } \quad \beta(z) \equiv \frac{1}{z}(\omega z-\omega+2)
$$

Proof. Since matrix $A$ fulfills the requirements of Theorem 1, the eigenvalues $\lambda$ of $L_{r, \omega}$ satisfy relationship (5.3) with $\mu$ being any eigenvalue of $L_{0,1}$. Equation (5.3) can be written as

$$
\lambda^{2}-\left[2(1-\omega)+r \omega \mu^{2}\right] \lambda+(\omega-1)^{2}+(r-\omega) \omega \mu^{2}=0 .
$$

A sufficient and necessary condition for the $M_{r, \omega}$-method to converge is that $\rho\left(L_{r, \omega}\right)$ $<1$ or equivalently the roots of $(5.4)$ be less than one in modulus, which according to Lemma 3, gives

$\left|(\omega-1)^{2}+(r-\omega) \omega \mu^{2}\right|<1, \quad\left|2(1-\omega)+r \omega \mu^{2}\right|<1+(\omega-1)^{2}+(r-\omega) \omega \mu^{2}$.

After some simple manipulation, the inequalities above are transformed into the equivalent ones below:

$$
\begin{aligned}
& \text { a. }-\left(1-\mu^{2}\right) \omega^{2}+2 \omega-2<r \omega \mu^{2}, \\
& \text { b. } r \omega \mu^{2}<-\left(1-\mu^{2}\right) \omega^{2}+2 \omega, \\
& \text { c. }-1 / 2\left(1-\mu^{2}\right) \omega^{2}+2 \omega-2<r \omega \mu^{2}, \\
& \text { d. } \mu^{2}<1 .
\end{aligned}
$$

Inequality (5.5d) gives one of the sufficient and necessary conditions for the $M_{r, \omega^{-}}$ method to converge, namely $\bar{\mu}<1$ or equivalently the $M_{0,1}$-method converges. Because of (5.5d), inequalities $(5.5 \mathrm{a}, \mathrm{b}, \mathrm{c})$ can be written as

$$
-1 / 2\left(1-\mu^{2}\right) \omega^{2}+2 \omega-2<r \omega \mu^{2}<-\left(1-\mu^{2}\right) \omega^{2}+2 \omega,
$$

which give $\left(1-\mu^{2}\right) \omega^{2}<4$ or equivalently

$$
-2 /\left(1-\underline{\mu}^{2}\right)^{1 / 2}<\omega_{\neq 0}<2 /\left(1-\underline{\mu}^{2}\right)^{1 / 2} .
$$

Having determined in this way all possible values for the overrelaxation parameter $\omega$ we now try to find, from (5.6), the corresponding values for $r$ by distinguishing two cases.

Case I: $\underline{\mu} \neq 0$. If $\omega>0$, then (5.6) can be written as

$$
\alpha(z) \equiv \frac{1}{\omega z}\left(\frac{1}{2} \omega^{2} z-\frac{1}{2} \omega^{2}+2 \omega-2\right)<r<\frac{1}{z}(\omega z-\omega+2) \equiv \beta(z),
$$

where we have set $z=\mu^{2}$. It is obvious that inequalities (5.8) will be satisfied for all values of $r$ such that

$$
\max _{z} \alpha(z)<r<\min _{z} \beta(z) .
$$

If, on the other hand, $\omega<0$, then (5.6) equivalent to $\beta(z)<r<\alpha(z)$; and these inequalities will be satisfied for all $r$ such that 


$$
\max _{z} \beta(z)<r<\min _{z} \alpha(z) .
$$

By studying the sign of the partial derivatives of $\alpha(z)$ and $\beta(z)$ with respect to $z$ we can easily construct Table 1 in which the behavior of these functions as $z$ increases from $\underline{\mu}^{2}$ to $\bar{\mu}^{2}$ and $\omega$ takes values from the corresponding intervals $I_{\omega}$ is presenied.

TABLE 1

\begin{tabular}{ccc}
\hline$I_{\omega}$ & $\alpha(z)$ & $\beta(z)$ \\
\hline$\left(-2 /\left(1-\underline{\mu}^{2}\right)^{1 / 2}, 0\right)$ & decreasing & decreasing \\
$(0,2]$ & increasing & decreasing \\
{$\left[2,2 /\left(1-\underline{\mu}^{2}\right)^{1 / 2}\right)$} & increasing & increasing \\
\hline
\end{tabular}

Taking now into consideration inequalities (5.9) and (5.10), we can easily obtain from Table 1 the ranges $I_{r}$ of the parameter $r$ for the corresponding ranges of $\omega$. These ranges are given in Table 2.

TABLE 2

\begin{tabular}{cc}
\hline$I_{\omega}$ & $I_{r}$ \\
\hline$\left(-2 /\left(1-\underline{\mu}^{2}\right)^{1 / 2}, 0\right)$ & $\left(\beta\left(\underline{\mu}^{2}\right), \alpha\left(\bar{\mu}^{2}\right)\right)^{*}$ \\
$(0,2]$ & $\left(\alpha\left(\overline{\mu^{2}}\right), \beta\left(\bar{\mu}^{2}\right)\right)$ \\
{$\left[2,2 /\left(1-\underline{\mu}^{2}\right)^{1 / 2}\right)$} & $\left(\alpha\left(\bar{\mu}^{2}\right), \beta\left(\underline{\mu}^{2}\right)\right)^{*}$ \\
\hline
\end{tabular}

*Note. It is obvious that the first and the third cases exist if and only if $\beta\left(\mu^{2}\right)<\alpha\left(\bar{\mu}^{2}\right)$ and $\alpha\left(\bar{\mu}^{2}\right)$ $<\beta\left(\underline{\mu}^{2}\right)$, respectively.

Case II: $\underline{\mu}=0$. Since inequalities (5.6) must be satisfied for both $\mu=0$ and $\mu \neq 0$ we have to distinguish two subcases. If $\mu=0$ relationships (5.6) give $0<\omega<$ 2 , while if $\mu \neq 0$, the analysis given in Case $I$ is valid and the possible values for $\omega$ and $r$ are given in Table 2. Since in this present case the values of $\omega$ and $r$ must satisfy (5.6) for all $\mu$, we easily conclude that their ranges are, respectively,

$$
I_{\omega} \equiv(0,2) \quad \text { and } \quad I_{r} \equiv\left(\alpha\left(\overline{\mu^{2}}\right), \beta\left(\overline{\mu^{2}}\right)\right) \text {. }
$$

This completes the proof of Theorem 2.

THEOREM 3. If $A$ is a consistently ordered matrix with nonvanishing diagonal elements, and if $L_{0,1}$ has real eigenvalues $\mu_{i} \mid i=1(1) N$ such that $0<\mu=\underline{\mu}=$ $\min _{i}\left|\mu_{i}\right|=\bar{\mu}=\max _{i}\left|\mu_{i}\right|<1$, then for $(r, \omega)=\left(2\left(1+\left(1-\mu^{2}\right)^{1 / 2}\right) / \mu^{2},-1 /\left(1-\mu^{2}\right)^{1 / 2}\right)$ or $\left(2 /\left(1+\left(1-\mu^{2}\right)^{1 / 2}\right), 1 /\left(1-\mu^{2}\right)^{1 / 2}\right), \rho\left(L_{r, \omega}\right)=0$.

Proof. By virtue of Lemma $1 \mu$ will be an eigenvalue of $L_{0,1}$. Since $\mu^{2}$ assumes one and only one fixed value we can derive values for $r \neq 0$ so that (5.1) has a double 
root. These values are

$$
r_{1}=2\left(1+\left(1-\mu^{2}\right)^{1 / 2}\right) / \mu^{2}, \quad r_{2}=2 /\left(1+\left(1-\mu^{2}\right)^{1 / 2}\right)
$$

and the double root for $\nu$ will be given by

$$
\nu=\left(2(1-r)+r^{2} \mu^{2}\right) / 2 .
$$

Since $\nu$ has only one value it is easy to determine $s$ (i.e. $\omega$ ) from (2.7) so that $\lambda=0$. For this we must have

$$
\omega=r /(1-\nu) .
$$

Thus from (5.11), (5.12) and (5.13) we finally obtain

$$
\omega_{1}=-1 /\left(1-\mu^{2}\right)^{1 / 2}, \quad \omega_{2}=1 /\left(1-\mu^{2}\right)^{1 / 2}
$$

The pairs $\left(r_{1}, \omega_{1}\right)$ and $\left(r_{2}, \omega_{2}\right)$ give $\rho\left(L_{r, \omega}\right)=0$ as is easily checked. Note. Since, as we have just seen, when $\underline{\mu}=\bar{\mu}, \rho\left(L_{r, \omega}\right)$ can be made zero, a value far better than the corresponding one is found for the optimum SOR which is $\rho\left(L_{\omega, \omega}\right)=$ $\left(\mu /\left(1+\left(1-\mu^{2}\right)^{1 / 2}\right)\right)^{2}$, it is strongly suggested that because of the continuity and at least in cases where $\underline{\mu}$ is very close to $\bar{\mu}$ we shall be able to find an optimum AOR method which will be better than the corresponding optimum SOR one.

Numerical Example. Let the following system (5.15) of two equations with two unknowns be given

$$
3 x_{1}-4 x_{2}=-1, \quad 2 x_{1}-3 x_{2}=-1
$$

We have

$$
A=\left[\begin{array}{ll}
3 & -4 \\
2 & -3
\end{array}\right], \quad x=\left[\begin{array}{l}
x_{1} \\
x_{2}
\end{array}\right], \quad b=\left[\begin{array}{l}
-1 \\
-1
\end{array}\right]
$$

and, therefore, by (2.2)

$$
D=\left[\begin{array}{cc}
3 & 0 \\
0 & -3
\end{array}\right], \quad A_{L}=\left[\begin{array}{cc}
0 & 0 \\
-2 & 0
\end{array}\right], \quad A_{U}=\left[\begin{array}{ll}
0 & 4 \\
0 & 0
\end{array}\right]
$$

Consequently, by using (2.7) we obtain

$$
L=\left[\begin{array}{cc}
0 & 0 \\
2 / 3 & 0
\end{array}\right], \quad U=\left[\begin{array}{cc}
0 & 4 / 3 \\
0 & 0
\end{array}\right], \quad c=\left[\begin{array}{c}
-1 / 3 \\
1 / 3
\end{array}\right] \quad \text { and } \quad L_{0,1}=\left[\begin{array}{cc}
0 & 4 / 3 \\
2 / 3 & 0
\end{array}\right] \text {, }
$$

whose eigenvalues are $\pm 2 \sqrt{2} / 3$. The $2 \times 2$ matrix $A$ is obviously a consistently ordered one, and the requirements of Theorem 3 are fulfilled. Since $\mu=2 \sqrt{2} / 3$ the optimum pairs $(r, \omega)$ are found from $(5.11)$ and $(5.14)$ to be $(3,-3)$ and $(3 / 2,3)$. The optimum AOR method corresponding to the first pair $(3,-3)$ is

$$
(I-3 L) x^{(n+1)}=(4 I-6 L-3 U) x^{(n)}-3 c \mid n=0,1,2, \ldots,
$$


while the one which corresponds to the pair $(3 / 2,3)$ is

$$
\left(I-\frac{3}{2} L\right) x^{(n+1)}=\left(-2 I+\frac{3}{2} L+3 U\right) x^{(n)}+3 c \mid n=0,1,2, \ldots .
$$

For both methods (5.16) and (5.17) $\rho\left(L_{r, \omega}\right)=0$ as is easily checked. The optimum SOR method corresponds to $\omega=2 /\left(1+\left(1-\mu^{2}\right)^{1 / 2}\right)=3 / 2$ and is, therefore,

$$
\left(I-\frac{3}{2} L\right) x^{(n+1)}=\left(-\frac{1}{2}+\frac{3}{2} U\right) x^{(n)}+\frac{3}{2} c \mid n=0,1,2, \ldots .
$$

For method (5.18) we can find $\rho\left(L_{\omega, \omega}\right)=1 / 2$. Using as the initial approximation to the solution $x$ of $(5.15)$ the vector $x^{(0)}=[00]^{T}$, we obtain from $(5.16) x^{(1)}=\left[\begin{array}{ll}1 & 1\end{array}\right]^{T}$ and $x^{(2)}=\left[\begin{array}{ll}1 & 1\end{array}\right]^{T}$. That is the exact solution after two iterations. By using (5.17) with $x^{(0)}=\left[\begin{array}{ll}0 & 0\end{array}\right]^{T}$ we obtain $x^{(1)}=\left[\begin{array}{ll}-1 & 0\end{array}\right]^{T}, x^{(2)}=\left[\begin{array}{ll}1 & 1\end{array}\right]$ and $x^{(3)}=\left[\begin{array}{ll}1 & 1\end{array}\right]^{T}$, that is, the exact solution after three iterations. In the same way for method (5.18) we obtain $x^{(26)}$ $=\left[\begin{array}{ll}0.9999988 & 0.9999993\end{array}\right]^{T}$ and $x^{(27)}=[0.99999920 .9999995]^{T}$, that is, the solution $x=\left[\begin{array}{lll}0.999999 & 1.000000\end{array}\right]^{T}$ correct to six decimal places after 27 iterations. In either scheme (5.16) or (5.17) we can find that we perform five multiplications and five additions and subtractions per iteration, while in scheme (5.18) we perform four multiplications and four additions and subtractions per iteration. Despite, however, the extra arithmetic per iteration needed in applying either scheme (5.16) or (5.17), the total number of calculations required to find the solution of system (5.15) correct to six decimal places by using the optimum AOR method is $(5 \times 2) /(4 \times 27) \times 100 \% \approx 9.3 \%$ and $(5 \times 3) /(4 \times 27) \times 100 \% \approx 13.9 \%$, respectively, of the corresponding total number of calculations required when using the optimum SOR method.

6. Final Remarks. As has been seen, the AOR or ESOR (for $r \neq 0$ ) method can be proved to be a very simple and powerful technique for solving linear systems of equations. Its powerfulness compared with the other well-known methods (e.g. the SOR method) lies in the fact that two parameters instead of usually at most one, are present. Full exploitation of the presence of these two parameters will provide us with methods which will converge faster than any other method of the same type. The determination of optimum acceleration and overrelaxation parameters is a matter which needs further investigation.

Acknowledgements. The author is most grateful to the referee for his valuable comments and suggestions especially for his suggestion that the AOR method, for $r \neq 0$, is the ESOR one.

\footnotetext{
Department of Mathematics

University of Ioannina

Ioannina, Greece
}

1. R. S. VARGA, Matrix Iterative Analysis, Prentice-Hall, Englewood Cliffs, N. J., 1962.

2. E. L. WACHSPRESS, Iterative Solution of Elliptic Systems and Applications to the Neutron Diffusion Equations of Reactor Physics, Prentice-Hall, Englewood Cliffs, N. J., 1966.

3. D. M. YOUNG, Iterative Solution of Large Linear Systems, Academic Press, New York and London, 1971. 